\title{
Entry of parainfluenza virus into cells as a target for interrupting childhood respiratory disease
}

\author{
Anne Moscona
}

Department of Pediatrics and Department of Microbiology and Immunology, Weill Medical College of Cornell University, New York, New York, USA.

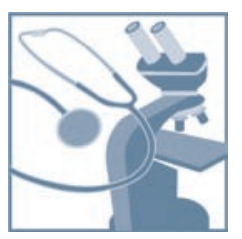

Human parainfluenza viruses cause several serious respiratory diseases in children for which there is no effective prevention or therapy. Parainfluenza viruses initiate infection by binding to cell surface receptors and then, via coordinated action of the 2 viral surface glycoproteins, fuse directly with the cell membrane to release the viral replication machinery into the host cell's cytoplasm. During this process, the receptor-binding molecule must trigger the viral fusion protein to mediate fusion and entry of the virus into a cell. This review explores the binding and entry into cells of parainfluenza virus type 3 , focusing on how the receptor-binding molecule triggers the fusion process. There are several steps during the process of binding, triggering, and fusion that are now understood at the molecular level, and each of these steps represents potential targets for interrupting infection.

\section{The paramyxovirus family of viruses and the parainfluenza viruses}

Viruses belonging to the paramyxovirus family, particularly respiratory syncytial virus (RSV), the recently identified human metapneumovirus (1), and the human parainfluenza viruses (HPIVs) types 1 , 2 , and 3 , cause the majority of childhood cases of croup, bronchiolitis, and pneumonia worldwide (2). HPIV3 alone is responsible for approximately $11 \%$ of pediatric respiratory hospitalizations in the US $(3,4)$ and is the predominant cause of croup in young infants, while HPIV1 and -2 tend to infect older children and adolescents. While other causes of respiratory disease in children - influenza and measles - have yielded in part to vaccination programs and antiviral therapy, children are still virtually unaided in their battle against the major causes of croup and bronchiolitis. RSV has been extensively studied, and some effective strategies of prophylaxis have been developed (5), but for the parainfluenza viruses, there are no therapeutic weapons; advances in preventing and treating diseases caused by both groups of viruses, especially the parainfluenza viruses, are far behind those in combatting diseases caused by many more genetically complex pathogens.

The parainfluenza viruses replicate in the epithelium of the upper respiratory tract and spread from there to the lower respiratory tract. Epithelial cells of the small airways become infected, and this is followed by the appearance of inflammatory infiltrates. The relationship among the tissue damage caused by the virus, the immune responses that help to clear the virus, and the inflammatory responses that contribute to disease is still quite enigmatic. Both humoral and cellular components of the immune system appear to contribute to both protection and pathogenesis $(6,7)$. Infection with HPIV in immunocompromised children (e.g., transplant recipients) is associated with a range of disease, from mild upper-respiratory symptoms to severe disease requiring mechanical ventilation and leading to death (8).

Nonstandard abbreviations used: F protein, viral fusion protein; 4-GU-DANA, 4-guanidino-Neu5Ac2en; HN, hemagglutinin-neuraminidase; HPIV, human parainfluenza virus; HR, heptad repeat; HR-C, C-terminal HR; HR-N, N-terminal HR; NA, neuraminidase protein; NDV, Newcastle disease virus; RSV, respiratory syncytial virus. Conflict of interest: The author has declared that no conflict of interest exists.

Citation for this article: J. Clin. Invest. 115:1688-1698 (2005).

doi:10.1172/JCI25669.
The hurdle for developing modes of preventing and treating croup and bronchiolitis caused by parainfluenza has been in large part a result of the gaps in our understanding of fundamental processes of viral biology and of the interaction of these viruses with their hosts during pathogenesis. For example, an inactivated HPIV1, $-2,-3$ vaccine used in infants in the late 1960 s was immunogenic but did not offer protection from infection $(9,10)$, which highlights the challenge of identifying which elements of the immune response confer protection from HPIVs. Primary infection with any HPIV does not confer permanent immunity against that virus, and repeated reinfection with the same agent within a year of the previous infection is common in young children. Immunity generated after the first infection is, however, often sufficient to restrict virus replication in the lower respiratory tract and prevent severe disease. Efforts are currently underway to develop live attenuated vaccines against HPIV1, -2 , and -3 , and an increased understanding of the molecular basis for attenuation of virulence may eventually lead to live HPIV vaccines that can be designed to be both attenuated and immunogenic and even to the development of combination respiratory virus vaccines (reviewed in ref. 11). Deeper understanding of the interplay among virusmediated pathology, beneficial immune responses, and exaggerated or disease-enhancing inflammatory responses will be vital for developing safe and effective vaccine strategies.

Antiviral therapy for the parainfluenza viruses has not been explored but, in light of the complexities involved in vaccination, could be a principal weapon against these diseases. Several features of the viral life cycle make these viruses vulnerable to attack. HPIVs enter their target cell by binding to a receptor molecule and then fusing their viral envelope with the cell membrane to gain admittance to the cytoplasm. Binding, fusion, and entry are therefore critical stages at which we could interfere with the viral life cycle and prevent disease. A firm grasp of the molecular mechanisms of these events is the basis for understanding respiratory disease pathogenesis and developing potential approaches to prevention and treatment.

\section{The parainfluenza virus life cycle}

HPIVs are members of the Respirovirus and Rubulavirus genera within the Paramyxoviridae family. The viruses are roughly spher- 


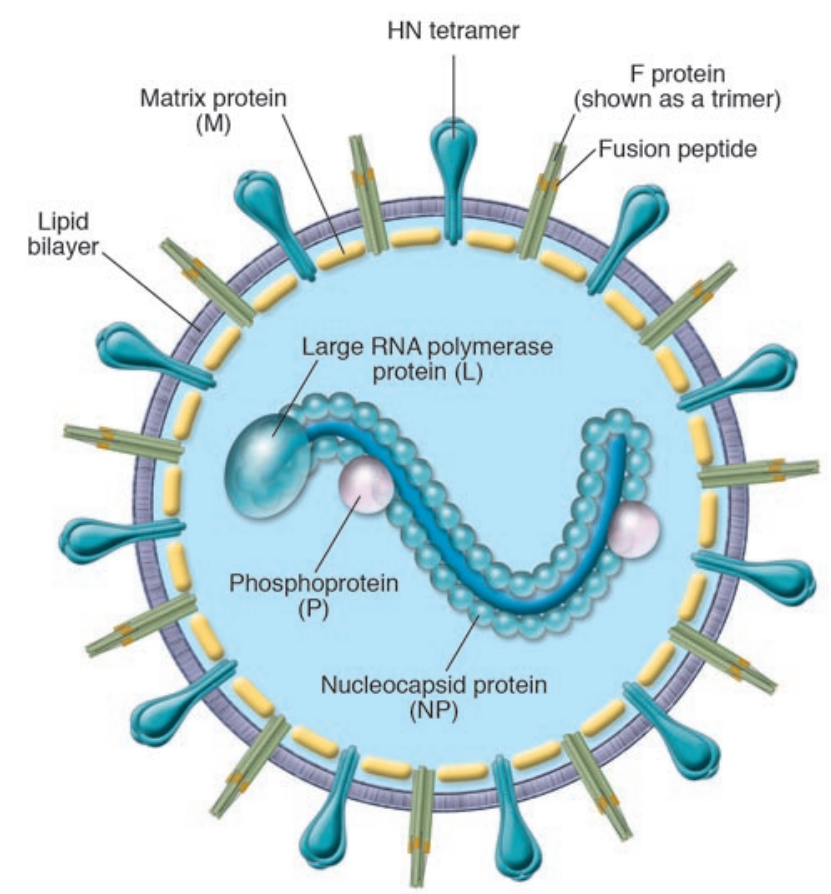

Figure 1

A schematic diagram of the parainfluenza virion. $L$, large RNA polymerase protein; M, matrix protein; NP, nucleocapsid protein; P, phosphoprotein. Modified with permission from New England Journal of Medicine (91).

ical in shape, approximately $150-400 \mathrm{~nm}$ in diameter, and have an envelope composed of host cell lipids and viral glycoproteins derived from the plasma membrane of the host cell during viral budding. The HPIV genome is single-stranded, negative-sense RNA that must be transcribed into message-sense RNA before it can be translated into protein. Like all negative-stranded RNA viruses, the HPIVs encode and package an RNA-dependent RNA polymerase in the virion particles (12). The RNA genome is approximately 15,500 nucleotides in length and is encapsidated by the viral nucleocapsid protein, forming helical nucleocapsids (Figure 1) (13).

The first step in infection of a cell by all HPIVs is binding to the target cell, via interaction of the viral receptor-binding molecule (hemagglutinin-neuraminidase [HN]) with sialic acid-containing receptor molecules on the cell surface (Figure 2). The viral envelope then is thought to fuse directly with the plasma membrane of the cell, mediated by the viral fusion protein (F protein), releasing the nucleocapsid into the cytoplasm $(14,15)$. The nucleocapsid released into the cytoplasm after fusion contains the genome RNA in tight association with the viral nucleocapsid protein, and this RNA/protein complex is the template both for transcription and for replication of the genome RNA that is packaged into progeny virions. The 6 viral genes encode the 2 surface glycoproteins HN and F; the matrix protein, which is involved in assembly and budding; the RNA polymerase proteins and a protein that encapsidates the RNA; and, through alternative reading frames and/or RNA editing, 1 or more proteins that are expressed only in the infected cell and whose roles include evasion of the host immune response.

Virions are formed, according to the prevailing model for virion assembly, when newly assembled nucleocapsids containing the full-length viral RNA genome along with the polymerase proteins bud out through areas of the plasma membrane that contain the
F and HN proteins and the matrix protein. In polarized epithelial cells, the viruses bud from the apical surface of the cell. The matrix protein binds to the nucleocapsid and also interacts with the cytoplasmic tails of the $\mathrm{HN}$ and $\mathrm{F}$ proteins, in this way mediating the alignment of the nucleocapsid with the areas of the plasma membrane containing viral glycoproteins in order to set the scenario for budding (16). The neuraminidase or receptor-cleaving activity of the HN molecule cleaves sialic acid-containing receptor moieties that would attach the viral HN protein to the cell surface and allows the release of newly budded particles from the cell to begin a new round of infection $(17,18)$.

\section{Role of the parainfluenza surface protein $\mathrm{HN}$ in receptor binding, receptor cleaving, and $F$ protein activation to mediate fusion}

The HN proteins of HPIVs are different from the receptor-binding glycoproteins of other members of the paramyxovirus family in that they possess both hemagglutinating (sialic acid-containing receptor-binding) and neuraminidase (sialic acid-containing receptor-cleaving) activities. The parainfluenza HN proteins are oriented such that their amino termini extend into the cytoplasm, while the $\mathrm{C}$ termini are extracellular (Figure 1). The $\mathrm{HN}$ protein is present on the cell surface and on the virion as a tetramer composed of disulfide-linked dimers (19). The molecule contains a cytoplasmic domain, a membrane-spanning region, a stalk region, and a globular head. Crystal structures of the HN protein of the avian paramyxovirus known as Newcastle disease virus (NDV) (20, 21) and more recently the HPIV3 HN protein (22) demonstrate that the globular head contains the primary sialic acid-binding site and the neuraminidase active site.

Far from simply serving to attach the virus to the surface of the cell and to release virus after replication, the interaction of the HN protein with its receptor is required for $\mathrm{F}$ protein-mediated membrane fusion during viral infection $(23,24)$. Studies of several related paramyxoviruses have revealed that, for most members of this family, the HN protein is essential to the F protein-mediated fusion process (25-27). While receptor binding is an important component of this process, attachment is not sufficient $(23,25,28)$; many F proteins demonstrate a requirement for the presence of an $\mathrm{HN}$ protein from the same type of virus (the homotypic HN protein) in order to mediate fusion $(25,26)$. One proposed explanation for this requirement is that the interaction between the $\mathrm{HN}$ and $\mathrm{F}$ proteins may be type specific and/or that a specific relationship between the structures and/or activities of the 2 proteins is required in order to maintain function (29). This final key function of the HN protein - promotion of fusion - has become amenable to mechanistic study only recently; upon binding to its receptor, parainfluenza $\mathrm{HN}$ protein plays a critical role in activating or "triggering" the F protein to assume its fusion-ready conformation $(30,31)$. Since insertion of the fusion peptide region of the F protein into the target cell membrane after the activation step is the key event leading to membrane fusion, efficiency of $\mathrm{F}$ protein triggering by the $\mathrm{HN}$ protein is an important variable influencing the extent of fusion mediated by the $\mathrm{F}$ protein and thus the extent of viral entry.

\section{Triggering of fusion during entry by enveloped viruses}

Entry of all enveloped viruses into host cells requires fusion of the viral and cell membranes. The fusion protein that mediates these processes differs among the enveloped viruses, but thus far these have been mechanistically grouped into just 2 classes of proteins. 


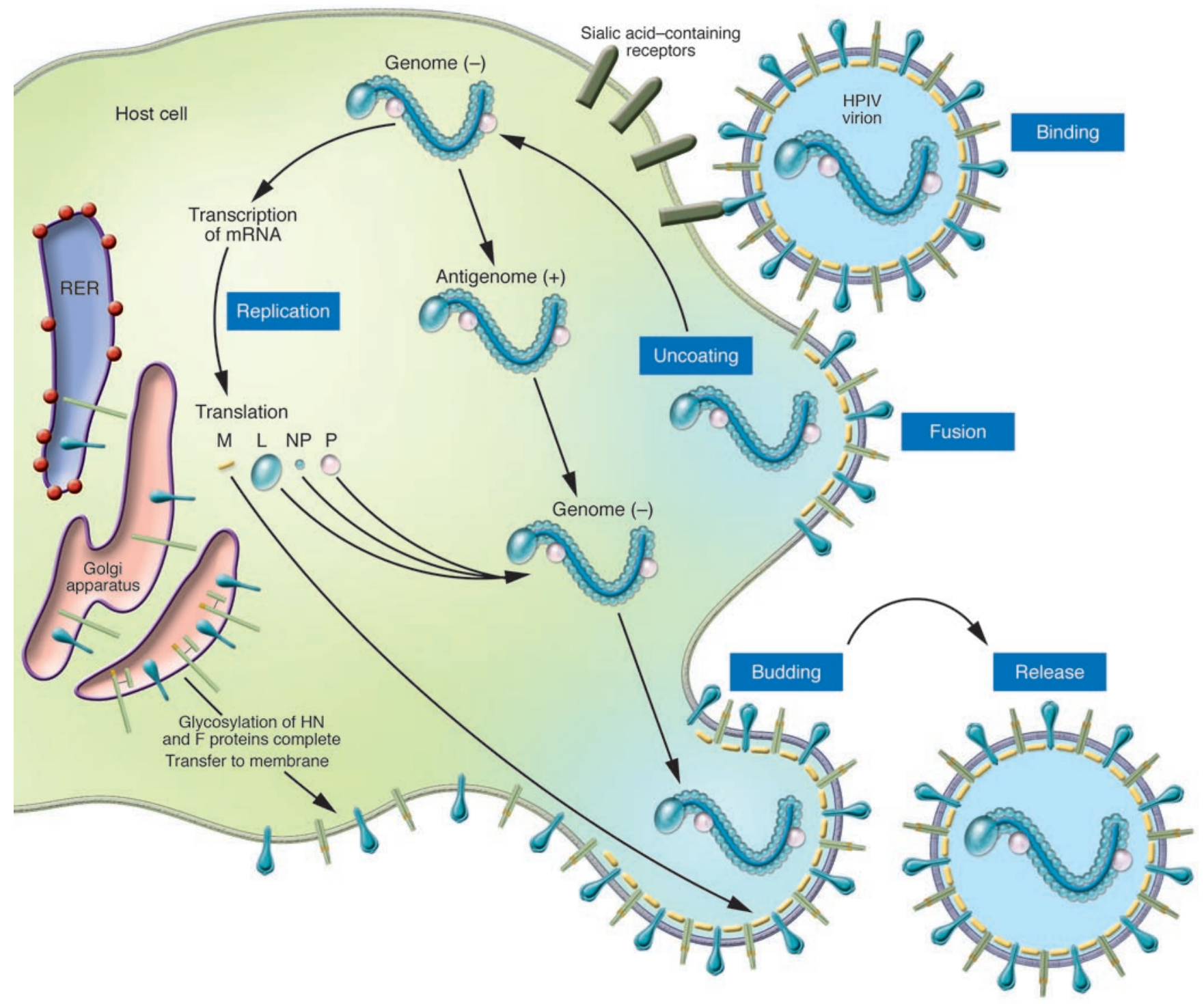

Figure 2

A schematic illustration of the parainfluenza viral life cycle. RER, rough endoplasmic reticulum.

The first, termed class I (reviewed in ref. 32), includes the paramyxovirus fusion proteins as well as the influenza hemagglutinin protein, the HIV gp120 fusion protein, and the Ebola virus fusion protein. Each is synthesized as a single polypeptide chain that forms trimers and is then cleaved by host proteases into 2 subunits, exposing a fusion peptide that will insert into the target cell membrane (33, 34) (reviewed in ref. 35). The trigger that initiates a series of conformational changes in the $\mathrm{F}$ protein leading to membrane merger differs depending on the pathway the virus uses to enter the cell and thus whether fusion needs to occur at the surface at neutral $\mathrm{pH}$ or in the endosome. The influenza HA protein has been the most extensively studied model for class I fusion (36), and the conformational change is triggered by the acidic $\mathrm{pH}$ of the endosome, which then allows the viral and endosomal membranes to fuse (35). Class II fusion proteins include the flavivirus dengue virus E protein (37), tick-borne encephalitis virus E protein (38), and togavirus Semliki Forest virus E1 protein (39), and despite pronounced differences in the structures of class I and class II fusion proteins, their transi- tion to the post-fusion state proceeds through structures similar enough to suggest a common mechanism (40).

The paramyxovirus fusion process is thought to occur at the surface of the target cell at neutral $\mathrm{pH}$, and activation of the $\mathrm{F}$ proteins occurs when the adjacent HN protein binds to the sialic acid-containing receptor, permitting fusion to occur. For HPIV3, the binding of $\mathrm{HN}$ protein to its receptor triggers the $\mathrm{F}$ protein to fuse with the target cell membrane, and alterations in the $\mathrm{HN}$ protein can alter its ability to trigger the F protein (30). The fusion peptides, which are buried within the F protein trimer, must be exposed in order to insert into the target cell membrane, and additional coreceptor binding events, for either the $\mathrm{HN}$ or F protein, have not been ruled out. New structural and experimental information about paramyxovirus $\mathrm{F}$ proteins has led to models for the structural transitions that occur during class I fusion (31, 32, 40-42) (Figure 3 ). The ectodomain of the membrane-anchored subunit of the F protein contains 2 hydrophobic domains, the fusion peptide and the transmembrane-spanning domain. Each of these domains is 
adjacent to 1 of 2 conserved heptad repeat (HR) regions: the fusion peptide is adjacent to the N-terminal HR (HR-N), and the transmembrane domain is adjacent to the $\mathrm{C}$-terminal HR (HR-C). These HR domains can oligomerize into coiled coils composed of several $\alpha$-helices. Once the F protein is activated, the fusion peptide inserts into the target membrane, first generating a transient "prehairpin" intermediate that is anchored to both viral and cell membranes (Figure 3, A-C). This form then refolds and assembles into a fusogenic 6-helix bundle structure as the HR-N and HR-C associate into a tight complex with $\mathrm{N}$ - and $\mathrm{C}$-peptides aligned in an antiparallel arrangement (Figure 3, D and E). The resultant helical coiled-coiled rods are located adjacent to the fusion peptides, forming a highly stable 6-helix bundle (Figure 3F). Refolding relocates the fusion peptides and transmembrane anchors to the same end of the coiled coil, bringing the viral and cell membranes together. The formation of a coiled-coil structure during this step generates the free energy for the membranes to bend toward each other and is thought to be the driving force for membrane fusion (31).

The role of the HN protein in activating the $\mathrm{F}$ protein has been explored using peptides that correspond to the HR domains of the F protein. Synthetic peptides derived from the HR regions of several paramyxovirus F proteins can inhibit fusion by binding to their complementary HR region and thereby preventing HR-N and HR-C from refolding into the stable 6-helix bundle structure required for fusion $(32,41)$. Susceptibility to inhibition by these peptides can be used as a gauge of F protein's progress through the steps outlined above, and such studies suggest that binding of the HN protein to a sialic acid-containing receptor induces a conformational change in the $\mathrm{F}$ protein (31). One model for the triggering of fusion suggests that, upon receptor binding, the $\mathrm{HN}$ protein itself undergoes a receptor-induced conformational change, which in turn triggers the conformational change in the F protein $(14,24)$. Results of experiments using $\mathrm{F}$ proteins that fuse without the HN protein suggest that the presence of the HN protein lowers the energy of activation required for F protein-mediated fusion (43). Many of the fundamental aspects of this fusion activation process remain to be understood; in particular, why is there a requirement for $\mathrm{HN}$-receptor binding in order to initiate fusion promotion? Does the HN protein undergo conformational change upon receptor binding? An understanding of how the $\mathrm{HN}$ protein carries out its $\mathrm{F}$ protein-triggering function is central to understanding paramyxovirus entry.

\section{Use of variant $\mathrm{HN}$ proteins to scrutinize the $\mathrm{HN}$-triggered F protein-mediated fusion process}

We have focused on the process whereby HPIV3 HN protein triggers the $\mathrm{F}$ protein after receptor interaction in order to understand this step in the entry of HPIV3 into the host cell. Since the HN protein has 3 functions (receptor binding, neuraminidase, and $\mathrm{F}$ protein activation) that each impact the fusion potential of the virus, the field had been hindered by the inability to study just 1 function - triggering - independently of the influence of other HN protein activities. A strategy for quantitating $\mathrm{F}$ protein triggering was developed using a panel of mutant $\operatorname{HN}$ proteins $(17,18,24,30)$ in order to map the 3 functions of the $\mathrm{HN}$ protein to specific regions of the protein. One of the mutant HN proteins displayed a defect in triggering the F protein, which demonstrated for the first time that $\mathrm{F}$ protein triggering is a distinct function of the $\mathrm{HN}$ protein (30). In our assays, insertion of $\mathrm{F}$ protein into target $\mathrm{rbc}$ membranes - or fusion between cells - served as a surrogate for the first steps of viral entry into target cells; while these systems differ from the setting of natural infection in the human lung, they allow representation of the events of $\mathrm{HN}$-receptor binding, F protein triggering, and $\mathrm{F}$ protein insertion, and mechanistic models can then be tested in either epithelial cell cultures or animal models.

Viruses with alterations in the HN protein that led to either an increased affinity of the protein for its receptor or decreased neuraminidase activity are more potent at inducing membrane fusion, which leads to the conclusion that it is the interaction of the $\mathrm{HN}$ protein with its receptor that is required for activation of F protein (29). HN proteins with either increased receptor affinity or decreased enzymatic (receptor-cleaving) activity stay engaged with their receptor longer than WT HN proteins (29). Both cell surface receptor-binding and neuraminidase activities of the HN protein, by impacting the duration of HN-receptor engagement, regulate $\mathrm{F}$ protein activation and fusion promotion. An intriguing mutant virus is one containing an alteration (P111S) in the stalk region of the $\mathrm{HN}$ molecule along with a globular head mutation that decreases neuraminidase activity. While viruses with this HN molecule are indeed neuraminidase-deficient, they are defective in promoting cell fusion, in contrast to what might be predicted (30). This was the first indication that there is a separate HN protein function that specifically triggers $F$ protein and that it resides in the stalk region of the protein.

The assay for measuring the F protein-triggering function of the $\mathrm{HN}$ protein (30) takes advantage of a sialic acid-containing receptor analog/neuraminidase inhibitor compound, 4-guanidino-Neu5Ac2en (4-GU-DANA; zanamivir, Relenza) that serves as a clinically effective antiinfluenza agent (44). While 4-GU-DANA inhibits HPIV neuraminidase activity, it does not prevent release of virus from the infected cell surface (45) as it does in the case of influenza viruses; instead, it blocks interaction between the parainfluenza HN protein and its receptor and thus - surprisingly - aids in the release of newly assembled virions from the infected cell (18). However, by interfering with $\mathrm{HN}$-receptor interaction, 4-GU-DANA blocks receptor binding and thereby blocks fusion and viral entry $(18,45)$. These findings have stimulated interest in designing binding/entry inhibitors for treatment of paramyxovirus infection.

When WT and variant HN proteins were compared for their ability to trigger WT F protein, several findings emerged. The HN protein derived from the neuraminidase-deficient/fusion-defective virus has 2 amino acid alterations (D216N, in the globular head, and P111S, in the stalk) and is slower at activating F protein than either the WT HN protein or the singly mutated D216N HN protein. Comparison with a singly mutated P111S HN protein revealed that this triggering delay is entirely attributable to the P111S mutation (30). F protein triggering was dramatically reduced by this change in the stalk region of the molecule, although there was no decrease in receptor binding avidity. Conducting the experiments at a temperature and $\mathrm{pH}$ not permissive of neuraminidase activity eliminated the effects of the HN protein's neuraminidase activity. As a result of the diminished triggering, cell fusion was also markedly reduced. The virus containing the doubly mutated D216N/P111S HN protein is the only paramyxovirus variant virus found to be specifically defective in the HN protein's F proteintriggering and fusion promotion function, and this was the first time that a fusion defect could be specifically attributed to the $\mathrm{HN}$ protein's triggering function, independent of the effects of the other $2 \mathrm{HN}$ protein functions (30). The fact that 1 change in the $\mathrm{HN}$ protein led to a specific defect in fusion promotion showed that it is, indeed, the HN protein that activates the F protein. 
A

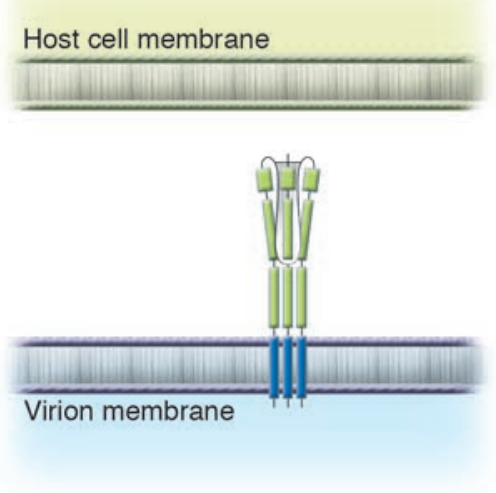

D

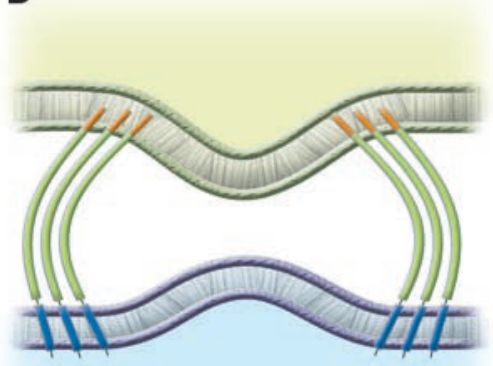

B

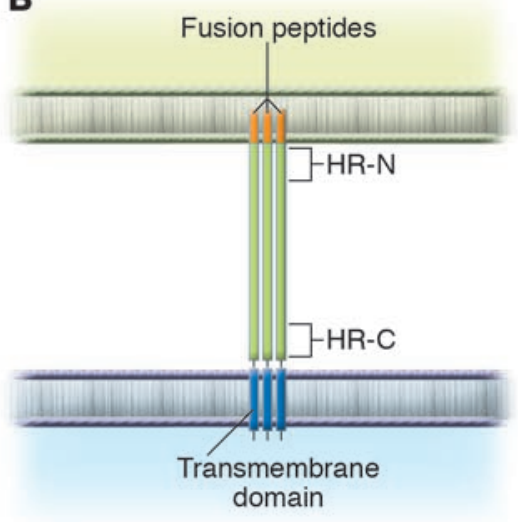

E

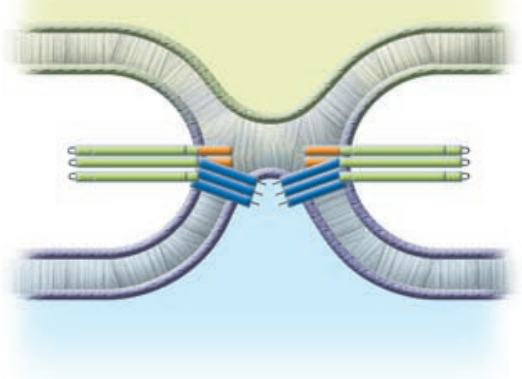

C

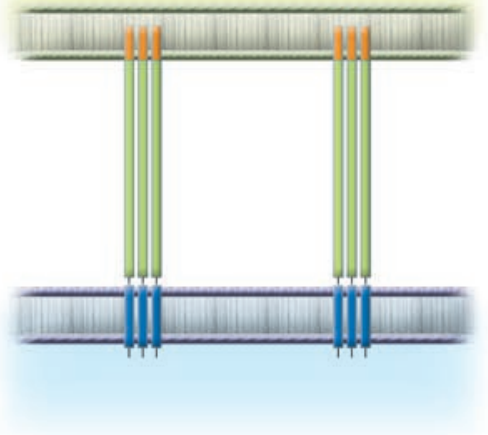

$\mathbf{F}$

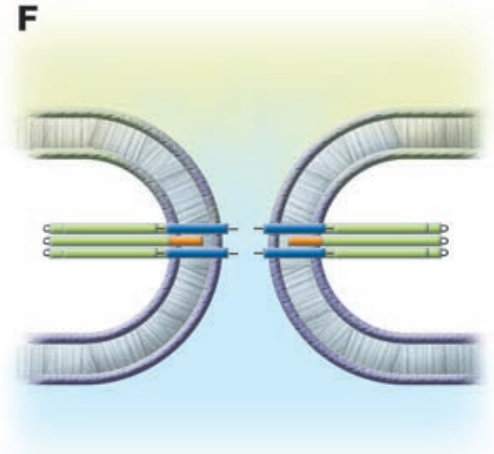

Figure 3

Model of class I fusion protein-mediated membrane fusion. (A and B) The trimeric paramyxovirus $F$ protein contains 2 hydrophobic domains: the fusion peptide and the transmembrane-spanning domain. Each is adjacent to 1 of 2 HR regions, HR-N and HR-C. (B) The F protein binds to a receptor on the host cell membrane, causing a conformational change and subsequent insertion of the hydrophobic fusion peptide into the host cell membrane. (C) Multiple F protein trimers are believed to mediate the fusion process. Protein refolding occurs when the host and viral cell membranes bend toward each other (D), and formation of a hemifusion stalk allows the lipids on the outer part of the membranes to interact (E). (F) When protein refolding is completed, the fusion peptide and the transmembrane domain are antiparallel in the same membrane, which creates the most stable form of the fusion protein. Figure modified with permission from Nature (40).

\section{Properties of the HN protein that modulate its ability to trigger the $\mathrm{F}$ protein}

When known variant $\mathrm{HN}$ proteins are studied under experimental conditions that allow assessment of all $3 \mathrm{HN}$ protein functions, it becomes evident that the balance among these properties determines entry (29). F protein triggering by the WT HN protein is dramatically reduced at a $\mathrm{pH}$ close to the optimum for neuraminidase ( $\mathrm{pH}$ 5.7); target cell receptors are released from $\mathrm{HN}$ protein by neuraminidase, and little triggering occurs. For the neuraminidase-dead $\mathrm{HN}$ protein (D216N/P111S HN), however, the rate and extent of F protein triggering are the same at both $\mathrm{pH} 5.7$ and $\mathrm{pH} 8.0(\mathrm{a} \mathrm{pH}$ at which the neuraminidase is not active), which confirms that for the WT HN protein, it is the enhanced neuraminidase activity at low $\mathrm{pH}$ that diminishes F protein triggering. Comparison of the doubly mutated protein with the P111S HN (30) revealed the effect of the HN protein's neuraminidase activity: the P111S HN (with residual neuraminidase activity) releases reversibly bound receptors from the HN protein, and unlike the D216N/P111S HN, its triggering cannot "catch up" by remaining in longer contact with receptor. A $\mathrm{pH}$ conducive to increased neuraminidase activity ( $\mathrm{pH}$ 5.7) completely abolishes triggering by the P111S HN. This comparison of different HN proteins - with neuraminidase activity as the only variable - illustrates the key role of this enzyme in regulating $\mathrm{F}$ protein triggering: neuraminidase reduces the chance that the HN protein will remain in contact with target cell receptors and thus prevents the slowly triggering HN protein from performing.

To assess the impact of receptor avidity on triggering, a variant HN (T193A) with higher avidity for receptor than the WT HN, and with WT neuraminidase activity, was useful. For this variant, $\mathrm{F}$ protein triggering remained as high at $\mathrm{pH} 5.7$ as at $\mathrm{pH}$ 8.0, and target cell receptor release remained as low at $\mathrm{pH} 5.7$ as at $\mathrm{pH}$ 8.0, which suggests that higher receptor avidity counterbalances the effect of increased neuraminidase. Both neuraminidase activity and receptor-binding avidity impact receptor availability and thereby the efficiency of the third function, $\mathrm{F}$ protein triggering. Thus, while mutations in the stalk region (e.g., P111S) affect the triggering potential of the HN protein, expression of this potential is also modulated by alterations in the globular head that affect $\mathrm{HN}$-receptor interaction. Triggering absolutely depends on HNreceptor interaction, and each of the 3 discrete properties of the $\mathrm{HN}$ protein independently affect the ability of the $\mathrm{HN}$ protein to complement the F protein in mediating fusion. 


\section{Probing the active sites on the HN protein}

Since HN-receptor interaction influenced by receptor avidity and neuraminidase activity in the globular head determines the possible extent of triggering, this domain would be a prime target for antiviral drugs and key to HN protein function. 4-GU-DANA blocks the receptor-binding and neuraminidase functions of the $\mathrm{HN}$ protein (44), and therefore variant $\mathrm{HN}$ proteins that are resistant to this inhibitor reveal information about the site or sites on the HN protein responsible for these functions.

Resistance to inhibitors at the binding site. Just when experimental data had allowed us to generate predictions about the mechanism of 4-GU-DANA resistance in HPIV3 as well as potential differences between HPIV3 and NDV, Lawrence et al. obtained the crystal structure of the HPIV3 HN protein (22). The structure of the globular head region is shown in Figure 4A complexed with sialic acid. A single alteration in the HN protein - T193I - leads to an HPIV3 variant with phenotypic resistance to the effects of 4-GUDANA in terms of both neuraminidase activity and receptor binding (45). Increased receptor-binding avidity alone can confer drug resistance and indeed accounts for part of the variant virus's 4-GUDANA-resistant properties (45). However, the T193I substitution does not confer resistance to a smaller molecule, DANA (identical to 4-GU-DANA except for a smaller substituent group at C4). In addition, substitution of a (smaller) alanine for the threonine in the active site (to generate T193A HN) does not confer resistance to 4-GU-DANA. It thus seemed possible that substitution of the larger isoleucine for threonine in the active site (at residue 193) might be excluding the inhibitor molecule from the active site of the resistant variant and contributing to resistance. Analysis of the crystal structure indeed shows that a T193I alteration in the HPIV3 HN protein would likely place the side chain of the isoleucine in a conformation that could lead to steric clash between the isoleucine at 193 and the guanidinium moiety of the 4-GUDANA. Figure 4B shows the active site region complexed with 4-GU-DANA and reveals the extension of the guanidine moiety into the pocket. The structure thus supports the notion that part of the resistance of the T193I HN variant to 4-GU-DANA indeed arises from a reduction in binding of 4-GU-DANA due to the bulk of the isoleucine side chain. This pathway whereby HPIV3 could develop resistance to such compounds is an issue that needs to be carefully considered in the design of antiviral analogs.

An alteration at the $H N$ protein dimer interface that affects avidity. One HPIV3 variant HN protein (H552Q HN) is resistant to 4-GUDANA solely due to its higher avidity for the receptor; this HN protein is like the WT HN protein in its neuraminidase activity and neuraminidase sensitivity to 4-GU-DANA. H552 lies at the HN dimeric interface (22) and does not appear to be involved in forming the primary receptor-binding site, which is consistent with the fact that the mutation has no effect on 4-GU-DANA binding affinity or on neuraminidase activity. How can the increased receptor-binding avidity of the H552Q variant $(24,45)$ be explained? Either the mutation causes an indirect conformational change at the binding site or $\mathrm{H} 552$ could represent part of a second receptorbinding site. The possible existence of a second binding site for the HPIV3 HN protein at or near the dimer interface is under study.

$H N$ molecules from related paramyxoviruses differ in their response to receptor analog inhibitors. The $3 \mathrm{D}$ crystal structure of the $\mathrm{HN}$ protein of the avian paramyxovirus NDV (20) suggests that one site could carry out both binding and neuraminidase activities, but researchers have postulated that an additional HN receptor-binding site exists (21), one exhibiting enzyme and receptor binding activity and the second exhibiting only receptor binding activity (46). In NDV, as in HPIV3, 4-GU-DANA drastically reduces infection. However, in the case of HPIV3, this is an effect mediated by the blocking of viral entry into the host cell, whereas in the case of NDV, the binding of the $\mathrm{HN}$ protein to its receptor is resistant to the inhibitory effects of 4-GU-DANA, which suggests that failure of progeny virion release due to neuraminidase inhibition by 4-GU-DANA accounts for the reduced infectivity for NDV (46). Thus, even 2 similar paramyxoviruses behave entirely differently in terms of their response to receptor analog inhibitors, a finding central to the discussion of antiviral approaches (discussed below).

The availability of the HPIV3 HN protein crystal structure and data from our mutant $\mathrm{HN}$ protein studies have allowed several correlations between HPIV3 HN structure and function to be made. Residue T193 forms part of the primary active site in the globular head of the HN protein, and alterations at this site can affect both receptor and inhibitor binding. H552 modulates avidity of the $\mathrm{HN}$ protein for its receptor but does not form part of the primary receptor-binding site, lying instead at the dimer interface. Residue D216 forms part of the framework of the globular head active site region, in line with the observation that it is key for neuraminidase activity (Figure 4A). Mutations at any of these sites may have the potential to alter fusion promotion, via alteration of the period of time that the HN protein and its receptor are in contact (which is essential for $\mathrm{F}$ protein activation), which emphasizes the relationship among the $3 \mathrm{HN}$ protein properties that contribute to entry.

\section{Contribution of $\mathrm{HN}$-receptor interaction to pathogenesis in vivo}

For HPIVs, the interplay among virus-mediated pathology, beneficial immune responses, and disease-enhancing inflammatory responses is not well understood, and it is likely that, as for RSV (47), in many cases, disease severity is increased and the pathology of clinical disease is actually caused by the inflammatory response rather than by the cytopathic effects of the virus (47). This fundamental concept is highlighted by the fact that virus titers in the infected host are generally waning by the time disease symptoms become apparent (2) and that virus titer does not correlate with the severity of lower-respiratory disease. A cotton rat model of disease has proven useful in initial analyses of the factors affecting the pathogenesis of HPIV3 in vivo. Experimental infection of the cotton rat leads to infection of bronchiolar epithelial cells and to bronchiolitis, mimicking human disease, which makes this a relevant model for HPIV3 lower-respiratory infection (48). In a study of cotton rats infected with WT HPIV3 and 3 of the variant HN viruses described above - HN T193A (high receptor avidity, globular head mutation), HN H552Q (high receptor avidity, dimer interface mutation), and HN D216N (low neuraminidase activity, globular head mutation) (49) - there was normal clearance of the variant viruses compared with WT viruses, the variant plaque morphology was preserved in vivo, and there was no reversion to WT phenotype in the infected animals. Quite surprisingly, each of the $\mathrm{HN}$ protein alterations led to striking differences in the ability of HPIV3 to cause extensive disease, and this effect was dissociated from effects on viral replication. The variants caused alveolitis and an interstitial infiltrate, while the WT virus only caused peribronchiolitis. The enhanced disease caused by the HN variants was manifested by greatly increased inflammatory cell infiltrate in the alveoli and interstitial spaces in the lung, characterized by notably 
A

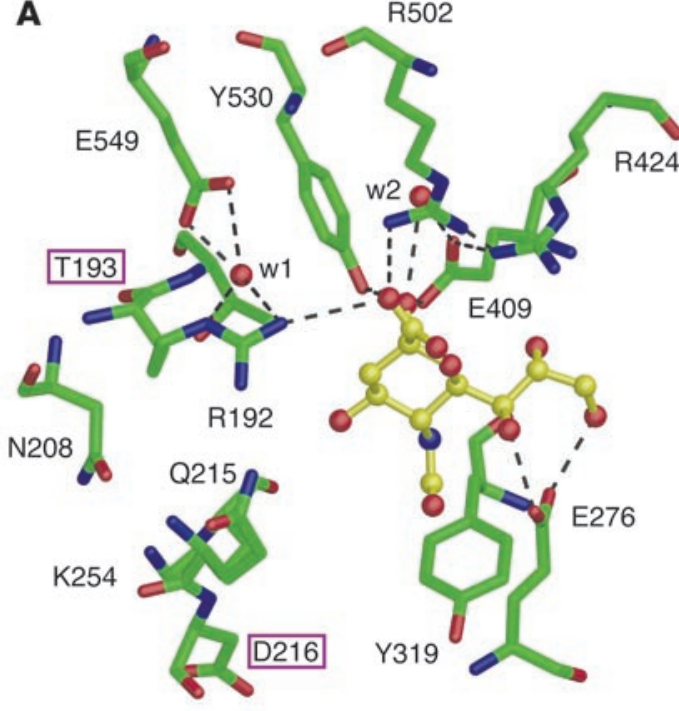

B

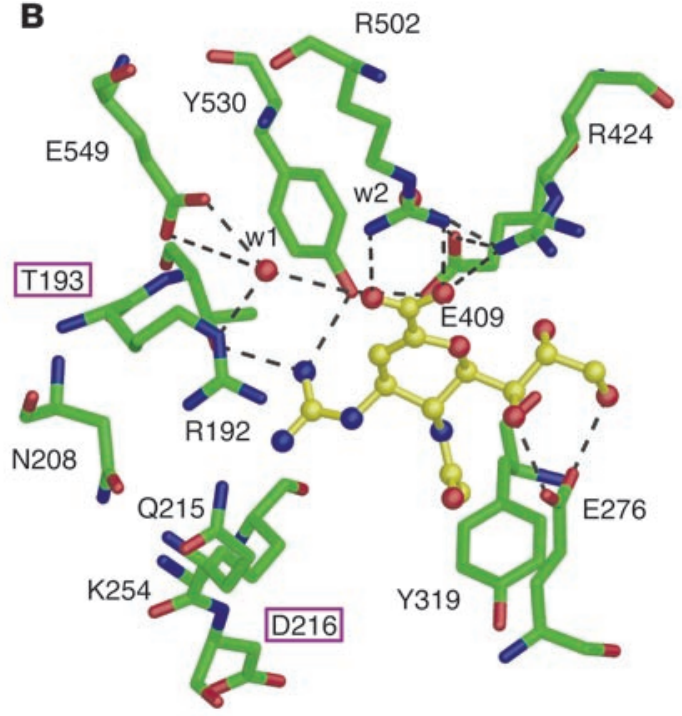

Figure 4

Images of the active site of the HPIV3 HN protein complexed with sialic acid (A) and 4-GU-DANA (zanamivir) (B) (both shown in yellow). Figure modified with permission from the Journal of Molecular Biology (22).

thickened alveolar walls and marked recruitment of inflammatory cells within the air spaces. These results suggest that these differences are indeed due to modulation of the inflammatory response through the different $\mathrm{HN}$ protein activity of the variants and are dissociated from viral replication or infectivity.

We hypothesize that changes in the HN protein that alter either the affinity of the HN protein for receptor or receptor-cleaving activity may alter the nature of the inflammatory response of the host. Using HN variants to dissect the etiology of enhanced disease, it may be possible to identify which component(s) of the immune system's response to HPIV3 contributes to disease. Indeed, preliminary experiments suggest that the enhanced pathology observed following infection of the cotton rat with HN-variant HPIVs correlates with specific alterations in the chemokine response to infection that are distinct for each variant $\mathrm{HN}$ protein (50). If further experiments support the finding that HN protein alterations specifically alter chemokine expression, this will provide information about the immune contribution to pathogenesis that can be used to develop therapies to modulate an overactive inflammatory response following HPIV3 infection.

For influenza, the severity of disease may be related to the ability of individual strains to induce proinflammatory cytokine expression $(51,52)$, and cytokine levels appear to correlate with severity of illness (53-55). In a mouse model of disease, it is the HA protein (receptor-binding protein) of the highly virulent 1918 influenza "Spanish flu" that confers the ability to cause severe disease; the disease (as in the cotton rat experiments described above) was widespread and involved recruitment of neutrophils to the alveoli, while viruses with WT HA protein led to only limited involvement of the alveoli, an effect not attributable to differences in replicative ability (56). These findings correlated with greatly enhanced cytokine production, which suggests that this specific HA protein is a critical determinant of macrophage activation and of production of neutrophil chemoattractants. These findings are reminiscent of the enhanced disease caused by the HPIV3 HN variants.

\section{Strategies for blocking fusion and viral entry}

Drawing on all that is known about entry into the cell by HPIV and other enveloped viruses, a number of potential strategies for influencing viral fusion become evident: first, blocking or perturbing F-triggering, and second, blocking $\mathrm{HN}$ protein-receptor binding. Both events would result in failure of the virus to enter the target cell. As mentioned earlier, peptides derived from the HR-N and HR-C regions of class I F proteins (called HR-N and HR-C peptides) can interfere with fusion intermediates of the F protein (41, 57-61). For example, the HIV envelope glycoprotein gp160 attaches to cellular receptors via its gp120 subunit and mediates fusion via its gp41 subunit; HIV peptides corresponding to the HR-C domain of gp41 are effective for treatment of HIV in humans, and T-20 was the first synthetic HR-C peptide approved for HIV treatment (62, 63). The C-terminus of the HR-N trimer contains a hydrophobic pocket that provides a potential binding site for small molecules that might interfere with the stability of the hairpin structure (64) and could provide advantages over peptides for clinical use. Intriguingly, a low-molecular-weight molecule that is highly effective in inhibiting RSV fusion was recently shown to bind within this hydrophobic pocket of HR-N, which suggests that indeed a small molecule that disrupts the hairpin can derail the fusion process (65); similar results have been obtained for the paramyxovirus simian virus 5, which suggests the general applicability of this approach (66). Inhibition of the F protein triggering process, by peptides or other small molecules that interact with the HR regions, is a promising area for development of antiviral therapies.

While F protein activation is key for entry, the correct timing of $\mathrm{F}$ protein activation is also essential; triggering must occur when the $\mathrm{F}$ protein is in contact with the target cell membrane. A fusion inhibitor effective against influenza was shown to prematurely trigger the conformational change in HA protein, rendering the virus incapable of fusion (67). A similar mechanism has been proposed for $\operatorname{HIV}(32,68)$. We suggest $(29)$ that correct timing of activation, which for HPIV3 must depend on the balance among the HN protein's receptor-binding, receptor-cleaving, and triggering 
activities, is critical to entry and represents a potential target for intervention. Swaying the balance of $\mathrm{HN}$ protein activities toward premature triggering of parainfluenza $F$ protein may be a strategy for preventing entry.

Since HN-receptor interaction is the critical prelude to F protein triggering, it is an attractive step for blocking viral entry, and the recent availability of 3D structures for NDV and HPIV3 would seem to make it more feasible to design inhibitors that specifically fit into the binding pocket on the globular head of the HN protein. For HPIV3, sialic acid analogs such as 4-GU-DANA, while they do inhibit neuraminidase (18), counteract infection by inhibiting receptor binding. It is thus possible that for HPIV3, sialic acid analogs may be viable antiviral agents by functioning as binding/entry inhibitors. For NDV, however, recent results (see above) indicate the opposite (46); these compounds inhibit neuraminidase and reduce infection in culture but do not completely prevent binding or block viral entry. It is possible that sialic acid analogs that are specifically designed to inhibit the active site of NDV neuraminidase may inhibit virion release as they do in the case of influenza virus (69). These data encourage optimism that receptor blockade may be effective for treating some paramyxoviruses and indeed for HPIV3. The data also highlight the fact that the paramyxoviruses differ from each other in terms of the properties of the HN protein in ways that bear on antiviral development and that each pathogen must be considered individually.

\section{Inhibition of neuraminidase: how HPIV is different from influenza and what can be learned from the influenza experience}

While neuraminidase inhibition does not seem a promising strategy for interfering with HPIV infection, this strategy has met with success in treating influenza virus infections. For influenza, HA protein, which recognizes the sialic acid moiety on the cell surface receptor, mediates both receptor binding of the virus to the cell and fusion of the viral envelope with the endosomal membrane; the neuraminidase protein (NA) is necessary for promoting the release of newly formed virions from the cell surface because it removes receptors for the virus, preventing self-aggregation (70). While in the case of HPIV infection, 4-GUDANA interferes with $\mathrm{HN}$-receptor interaction and thus actually enhances virus release (18), in the case of influenza virus infection, the clinical effectiveness of this molecule has been attributed to its ability to halt spread of the virus when given early in infection $(71,72)$. We have found that sialic acid-based inhibitors of influenza virus NA can also exert a direct effect on the function of the other envelope protein, HA protein (44). Recent experiments in primary cultures of human airway epithelium cells demonstrated that oseltamivir (a sialic acid analog related to 4-GU-DANA, discussed below) interfered with influenza infection at the early stage of entry (73). Thus, while the effects of 4-GU-DANA on influenza virus have been ascribed purely to the prevention of viral release by neuraminidase inhibition, these results suggested that the antiviral mechanism of action of 4-GU-DANA might be broader and may extend to interfering with viral entry (44). It will be of great interest to determine whether, as is possible for HPIV, neuraminidase may play a role in early infection and whether inhibition at this stage of the viral life cycle contributes to clinical effectiveness.

The design of 4-GU-DANA as a sialic acid analog antiviral compound that mimics the virus's natural substrate proceeded directly from 3D structural studies of the influenza NA (69). Zanamivir is administered by oral inhalation, which delivers the drug directly to the respiratory tract epithelium, and is clinically effective if given early in infection, with remarkably few side effects (74). Shortly after the introduction of zanamivir in clinical practice, an orally available NA inhibitor, oseltamivir, was developed. The NA inhibitors as a class are effective against all NA subtypes and therefore against all strains of influenza (74-82), including the 2004 avian influenza H5N1 strains that are resistant to the M2 inhibitors $(83,84)$.

An important aspect of the utility of these compounds is that until recently, there seemed to be very little development of resistance to neuraminidase inhibitors (85). The structure-based design of the neuraminidase inhibitors contributed to the fact that it is unlikely for the viral neuraminidase to change in such a way as to confer resistance, while still maintaining function. The neuraminidase inhibitors must fit directly into the enzyme's active site pocket in order to block the enzyme's activity, and since zanamivir was designed to closely resemble the natural substrate, mutations that interfere with zanamivir binding rarely permit enzyme function. In vitro experiments (86) timed the emergence of mutations conferring resistance to several of the then-new neuraminidase inhibitors. The rapidity with which the virus developed resistance to each compound was directly related to how different the inhibitor molecule was from the structure of the natural substrate. This led to the idea that the closer the drug structure is to that of the natural substrate, the less likely it is that the neuraminidase can mutate and maintain function (87); oseltamivir has a variety of modifications from the natural substrate, and hence resistance was considered more likely to develop to this drug than to zanamivir.

It seems plausible now that both the optimistic predictions for zanamivir and the concerns raised about oseltamivir's design are being borne out in clinical practice. For zanamivir, no resistant virus has been isolated after treatment of immunocompetent people, but for oseltamivir, the frequency of post-treatment neuraminidase resistance is higher. While only about $0.4 \%$ of treated adults harbored influenza viruses with oseltamivir-resistant neuraminidases, this number rose to at least $4 \%$ for treated children. Recently, a small study of children in Japan (88) found that, out of 50 children treated with oseltamivir, 9 (18\%) harbored viruses with drug-resistance mutations in the neuraminidase gene; the mutations were located where predicted from the in vitro studies discussed above. These mutations occurred far more frequently than has been previously observed (89), but it has not yet been established whether this is a general phenomenon, nor whether the oseltamivir-resistant viruses are transmissible or pathogenic (90).

This tale, both in the elements of resounding success and in the elements of increasing concern about the development of resistance (88), points to the great utility of structural and in vitro studies brought to bear on development of antiviral therapies. In order to continue to benefit from these potent antiviral compounds, it is critical to understand more about which features of neuraminidase inhibitors will discourage the emergence of resistance (90). By understanding the structural basis of resistance, it ought to be possible to design effective neuraminidase inhibitors that are less likely to select for resistant neuraminidase molecules. The same principles should hold true for designing molecules to interact with the sites on the parainfluenza HN protein that participate in receptor binding and $\mathrm{F}$ protein triggering. 


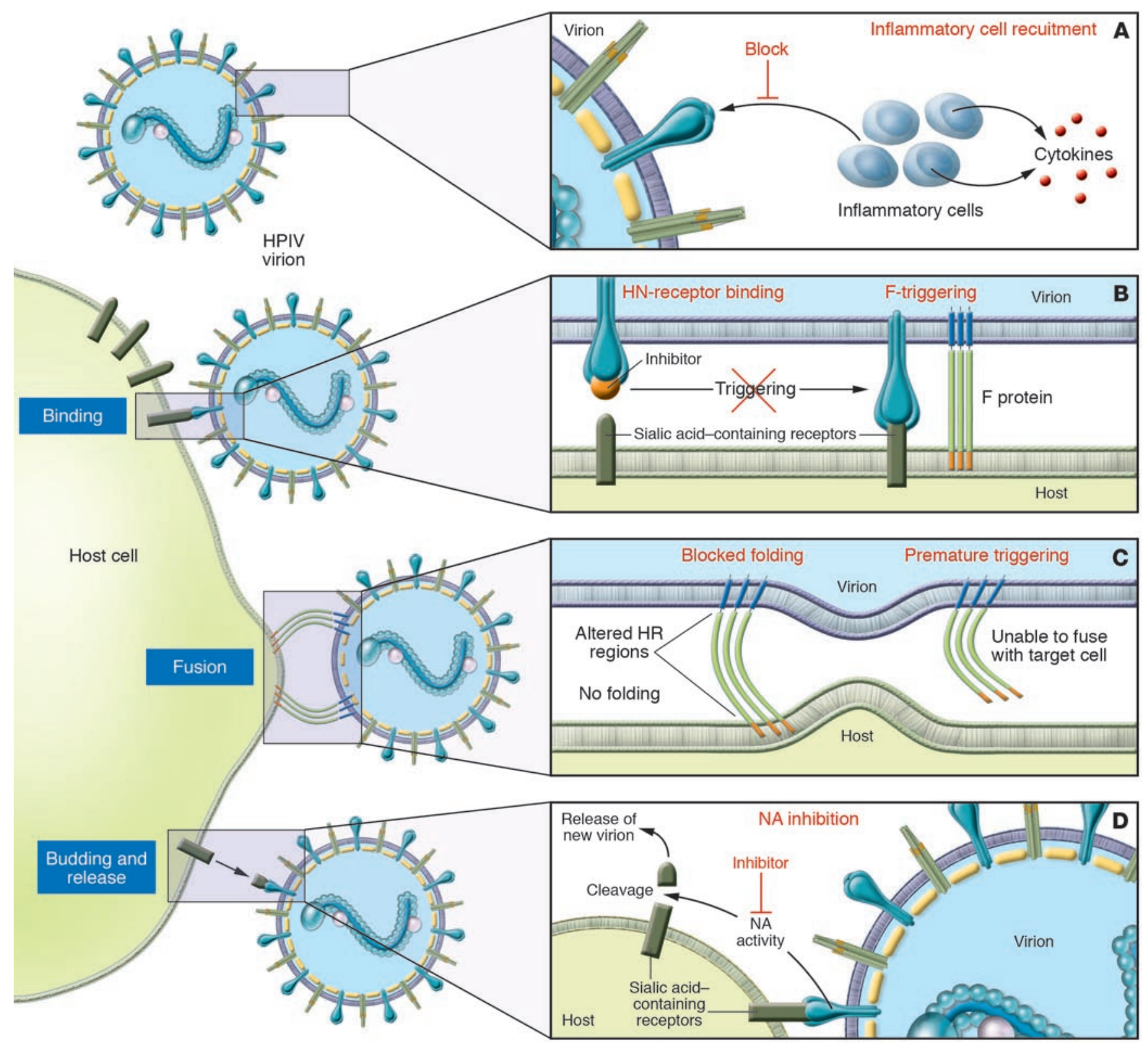

Figure 5

Sites or steps within the viral life cycle that represent potential targets for antiviral molecules. (A) Agents directed at blocking the ability of the HN protein to recruit inflammatory cells to the lung and subsequent cytokine expression may reduce the inflammatory response to the $\mathrm{HN}$ protein and ameliorate disease. (B) Molecules that fit into the binding pocket on the HN globular head may inhibit HN-receptor binding and the subsequent F protein triggering action of the HN protein stalk. On the left, the HN protein is shown bound with an inhibitor, precluding the scenario shown on the right, in which the HN protein's binding to the cell has led to its activation of F protein. (C) F protein peptides may be designed to prevent the refolding event that is essential to fusion during virus entry into the host cell. In addition, the F protein could perhaps be prematurely triggered and become incapacitated before it reaches the target host cell membrane. (D) Finally, the HN protein has NA activity and thus the ability to cleave the sialic acid moieties of the cellular receptors, promoting the release of new virions from the host cell surface. Specific inhibition of this activity may prevent virion entry into additional uninfected cells.

\section{Using what we know to block parainfluenza virus pathogenesis, and learning more}

The multiple roles of the parainfluenza envelope glycoproteins lend themselves to potential strategies for interfering with viral entry, pathogenesis, and disease (Figure 5). Since parainfluenza pathogenesis is likely due in large part to the exuberant inflammatory response to infection, the finding that specific alterations in the HN protein correlate with enhanced pathology, possibly due to the HN protein's role in induction of inflammatory responses, suggests approaches to modulate this inflammatory response and ameliorate disease (Figure 5A). Given the key role of the inflammatory response as well as the facts that viral replication in the respiratory tract peaks soon after disease onset and that viral titers do not correlate directly with disease severity, any antiviral strategy 
that targets early steps in the life cycle and thus only ameliorates illness at the stage of viral replication would need to be administered as early during replication as possible. For direct prevention or debilitation of the infection process, the most obvious avenue is that of interfering with receptor binding. Now that the $3 \mathrm{D}$ crystal structure of the HN protein is available, binding inhibitors can be designed specifically to fit into the binding pocket on the globular head of the HN protein (Figure 5B). In addition, this blockade will interfere with the F protein triggering function of the HN protein, which can only occur when the HN protein is in contact with its receptor. The F protein triggering function itself provides an exciting range of strategies for future exploration. First, peptides corresponding to the HR repeats of the F protein can be designed to prevent the $\mathrm{F}$ protein from reaching the state at which it can mediate fusion of the viral envelope with the host cell (Figure 5C). Preliminary studies suggest that it may even be possible to prematurely trigger the $\mathrm{F}$ protein, incapacitating it before it can reach its target. While specific mutations in the stalk region can influence the ability of the HN protein to trigger the F protein, and specific features of the globular head modulate this triggering, it remains completely unknown how the "activating signal" is transmitted from the $\mathrm{HN}$ protein to the F protein. If receptor binding induces a conformational change in the HN protein, how is this change communicated to lead to triggering of the F protein? Finally, the $\mathrm{HN}$ protein possesses neuraminidase activity and thus the ability to cleave the sialic acid moieties of the cellular receptors, promoting the release of new virions from the host cell surface. Specific inhibition of this activity may prevent virion entry into additional uninfected cells (Figure 5D). These potential therapeutic targets are now being actively pursued and promise to open new avenues for interfering with infection by HPIVs and other viruses.

\section{Acknowledgments}

Support has been generously provided to the author's laboratory for the work discussed here by US Public Health Service Grant AI31971. The author would like to thank the present and past laboratory members and colleagues for their creative contributions to the field and in particular to gratefully acknowledge Matteo Porotto, Richard W. Peluso, Olga Greengard, Gregory Prince, Robert A. Lamb, Ruth A. Karron, Kurt Hirschhorn, and Carol Heilman.

Address correspondence to: Anne Moscona, Department of Pediatrics and Department of Microbiology and Immunology, Weill Medical College of Cornell University, 515 East 71st Street, 600D, New York, New York 10021, USA. Phone: (212) 746-4523; Fax: (212) 746-8117; E-mail:anm2047@med.cornell.edu.
1. Williams, J.V., et al. 2004. Human metapneumovirus and lower respiratory tract disease in otherwise healthy infants and children. N. Engl. J. Med. 350:443-450.

2. Collins, P., Chanock, R., and McIntosh, K. 1996. Parainfluenza viruses. In Fields virology. B. Fields et al., editors. Lippincott-Raven Publishers. Philadelphia, Pennsylvania, USA. 1205-1241.

3. Chanock, R.M. 1990. Control of pediatric viral diseases: past successes and future prospects [review]. Pediatr. Res. 27(Suppl.):S39-S43.

4. Murphy, B.R. 1988. Current approaches to the development of vaccines effective against parainfluenza viruses [review]. Bull. World Health Organ. 66:391-397.

5. Groothuis, J., et al. 1993. Prophylactic administration of respiratory syncytial virus immune globulin to high-risk infants and young children. N. Engl. J. Med. 329:1524-1530.

6. Smith, C., Purcell, R., Bellanti, J., and Chanock, R. 1966. Protective effect of antibody to parainfluenza type 1 virus. N. Eng. J. Med. 275:1145-1152.

7. Tremonti, L., Lin, J., and Jackson, G. 1968. Neutralizing activity in nasal secretions and serum in resistance of volunteers to parainfluenza virus type 2 . J. Immunol. 101:572-577.

8. Apalsch, A.M., Green, M., Ledesma-Medina, J., Nour, B., and Wald, E.R. 1995. Parainfluenza and influenza virus infections in pediatric organ transplant recipients. Clin. Infect. Dis. 20:394-399.

9. Chin, J., Magoffin, R., Shearer, L., Schieble, J., and Lennette, E.1969. Field evaluation of a respiratory syncytial virus vaccine and a trivalent parainfluenza virus vaccine in a pediatric population. Am.J. Epidemiol. 89:449-463.

10. Fulginiti, V., et al., 1969. Respiratory virus immunization. I. A field trial of two inactivated respiratory virus vaccines; an aqueous trivalent parainfluenza virus vaccine and an alum-precipitated respiratory syncytial virus vaccine. Am. J. Epidemiol. 89:435-448.

11. Durbin, A.P., and Karron, R.A. 2003. Progress in the development of respiratory syncytial virus and parainfluenza virus vaccines. Clin. Infect. Dis. 37:1668-1677.

12. Lamb, R.A., Mahy, B.W., and Choppin, P.W. 1976. The synthesis of sendai virus polypeptides in infected cells. Virology. 69:116-131.

13. Choppin, P.W., and Compans, R. 1975. Reproduc- tion of paramyxoviruses. In Comprehensive virology. R.R. Wagner, editor. Plenum Press. New York, New York, USA. 95-178.

14. Lamb, R. 1993. Paramyxovirus fusion: a hypothesis for changes [review]. Virology. 197:1-11.

15. Plemper, R.K., Lakdawala, A.S., Gernert, K.M., Snyder, J.P., and Compans, R.W. 2003. Structural features of paramyxovirus $F$ protein required for fusion initiation. Biochemistry. 42:6645-6655.

16. Ali, A., and Nayak, D.P. 2000. Assembly of Sendai virus: $M$ protein interacts with $F$ and $H N$ proteins and with the cytoplasmic tail and transmembrane domain of F protein. Virology. 276:289-303.

17. Huberman, K., Peluso, R., and Moscona, A. 1995. The hemagglutinin-neuraminidase of human parainfluenza virus type 3: role of the neuraminidase in the viral life cycle. Virology. 214:294-300.

18. Porotto, M., Greengard, O., Poltoratskaia, N. Horga, M.-A., and Moscona, A. 2001. Human parainfluenza virus type $3 \mathrm{HN}$-receptor interaction: the effect of 4-GU-DANA on a neuraminidase-deficient variant. J. Virology. 76:7481-7488.

19. Russell, R., Paterson, R., and Lamb, R. 1994. Studies with cross-linking reagents on the oligomeric form of the paramyxovirus fusion protein. Virology. 199:160-168.

20. Crennell, S., Takimoto, T., Portner, A., and Taylor, G. 2000. Crystal structure of the multifunctional paramyxovirus hemagglutinin-neuraminidase. Nat. Struct. Biol. 7:1068-1074.

21. Zaitsev, V., et al. 2004. Second sialic acid binding site in Newcastle disease virus hemagglutininneuraminidase: implications for fusion. J. Virol. 78:3733-3741.

22. Lawrence, M.C., et al. 2004. Structure of the haemagglutinin-neuraminidase from human parainfluenza virus type III. J. Mol. Biol. 335:1343-1357.

23. Moscona, A., and Peluso, R.W. 1991. Fusion properties of cells persistently infected with human parainfluenza virus type 3 : participation of hemagglutinin-neuraminidase in membrane fusion. J. Virol. 65:2773-2777.

24. Moscona, A., and Peluso, R.W. 1993. Relative affinity of the human parainfluenza virus 3 hemagglutinin-neuraminidase for sialic acid correlates with virus-induced fusion activity. J. Virol. 67:6463-6468. 25. Hu, X., Ray, R., and Compans, R.W. 1992. Func- tional interactions between the fusion protein and hemagglutinin-neuraminidase of human parainfluenza viruses. J. Virol. 66:1528-1534.

26. Horvath, C.M., Paterson, R.G., Shaughnessy, M.A., Wood, R., and Lamb, R.A. 1992. Biological activity of paramyxovirus fusion proteins: factors influencing formation of syncytia. J. Virol. 66:4564-4569.

27. Bagai, S., and Lamb, R. 1995. Quantitative measurement of paramyxovirus fusion: differences in requirements of glycoproteins between SV5 and human parainfluenza virus 3 or Newcastle disease virus. J. Virol. 69:6712-6719.

28. Sergel, T., McGinnes, L.W., Peeples, M.E., and Morrison, T.G. 1993. The attachment function of the Newcastle disease virus hemagglutinin-neuraminidase protein can be separated from fusion promotion by mutation. Virology. 193:717-726.

29. Porotto, M., Murrell, M., Greengard, O., Doctor, L., and Moscona, A. 2005. Influence of the human parainfluenza virus 3 attachment protein's neuraminidase activity on its capacity to activate the fusion protein. J. Virol. 79:2383-2392.

30. Porotto, M., Murrell, M., Greengard, O., and Moscona, A. 2003. Triggering of human parainfluenza virus 3 fusion protein (F) by the hemagglutinin-neuraminidase (HN): an $\mathrm{HN}$ mutation diminishing the rate of $\mathrm{F}$ activation and fusion. J. Virol. 77:3647-3654

31. Russell, C.J., Jardetzky, T.S., and Lamb, R.A. 2001. Membrane fusion machines of paramyxoviruses: capture of intermediates of fusion. $E M B O \mathrm{~J}$. 20:4024-4034.

32. Colman, P.M., and Lawrence, M.C. 2003. The structural biology of type I viral membrane fusion. Nat. Rev. Mol. Cell Biol. 4:309-319.

33. Scheid, A., and Choppin, P. 1974. Identification of biological activities of paramyxovirus glycoproteins. Activation of cell fusion, hemolysis and infectivity by proteolytic cleavage of an inactive protein of Sendai virus. Virology. 57:470-490.

34. Homma, M., and Ouchi, M. 1973. Trypsin action on the growth of Sendai virus in tissue culture cells. Structural difference of Sendai viruses grown in eggs and tissue culture cells. J. Virol. 12:1457-1465.

35. Hernandez, L.D., Hoffman, L.R., Wolfsberg, T.G., and White, J.M. 1996. Virus-cell and cell-cell fusion. Annu. Rev. Cell Dev. Biol. 12:627-661. 
36. Skehel, J.J., and Wiley, D.C. 2000. Receptor binding and membrane fusion in virus entry: the influenza hemagglutinin. Annu. Rev. Biochem. 69:531-569.

37. Modis, Y., Ogata, S., Clements, D., and Harrison, S.C. 2004. Structure of the dengue virus envelope protein after membrane fusion. Nature. 427:313-319.

38. Bressanelli, S., et al. 2004. Structure of a flavivirus envelope glycoprotein in its low-pH-induced membrane fusion conformation. EMBO J. 23:728-738.

39. Gibbons, D.L., et al. 2004. Conformational change and protein-protein interactions of the fusion protein of Semliki Forest virus. Nature. 427:320-325.

40. Jardetzky, T.S., and Lamb, R.A. 2004. Virology: a class act. Nature. 427:307-308

41. Baker, K.A., Dutch, R.E., Lamb, R.A., and Jardetzky, T.S. 1999. Structural basis for paramyxovirusmediated membrane fusion. Mol. Cell. 3:309-319.

42. Dutch, R.E., Jardetzky, T.S., and Lamb, R.A. 2000. Virus membrane fusion proteins: biological machines that undergo a metamorphosis. Biosci. Rep. 20:597-612.

43. Paterson, R.G., Russell, C.J., and Lamb, R.A. 2000 Fusion protein of the paramyxovirus SV5: destabilizing and stabilizing mutants of fusion activation. Virology. 270:17-30.

44. Greengard, O., Poltoratskaia, N., Leikina, E., Zimmerberg, J., and Moscona, A. 2000. The anti-influenza virus agent 4-GU-DANA (zanamivir) inhibits cell fusion mediated by human parainfluenza virus and influenza virus HA. J. Virol. 74:11108-11114.

45. Murrell, M., Porotto, M., Weber, T., Greengard, O, and Moscona, A. 2003. Mutations in human parainfluenza virus type $3 \mathrm{HN}$ causing increased receptor binding activity and resistance to the transition state sialic acid analog 4-GU-DANA (zanamivir). J. Virol. 77:309-317.

46. Porotto, M., et al. 2004. Inhibition of parainfluenza type 3 and Newcastle disease virus hemagglutininneuraminidase receptor binding: effect of receptor avidity and steric hindrance at the inhibitor binding sites. J. Virol. 78:13911-13919.

47. Openshaw, P.J., Culley, F.J., and Olszewska, W. 2001. Immunopathogenesis of vaccine-enhanced RSV disease. Vaccine. 20(Suppl. 1):S27-S31.

48. Porter, D., Prince, G., Hemming, V., and Porter, H. 1991. Pathogenesis of human parainfluenza virus 3 infection in two species of cotton rat: Sigmodon hispidus develops bronchiolitis, while Sigmodon fulviventer develops interstitial pneumonia. J. Virol. 65:103-111.

49. Prince, G.A., Ottolini, M.G., and Moscona, A. 2001. Contribution of the human parainfluenza virus type $3 \mathrm{HN}$-receptor interaction to pathogenesis in vivo. J. Virol. 75:12446-12451.

50. Eichelberger, M., Bauchiero, S., Ottolini, M., Prince, G., and Moscona, A. 2003. Individual mutations that alter the hemagglutinin-neuraminidase molecule result in distinct alterations in the inflammatory response to human parainfluenza 3 viruses. In Abstracts of papers presented at the 2003 Meeting on Molecular Approaches to Vaccine Design. Cold Spring Harbor Laboratory. Cold Spring Harbor, New York, USA. 21.

51. Guan, Y., et al. 2004. H5N1 influenza: a protean pandemic threat. Proc. Natl. Acad. Sci. U. S. A. 101:8156-8161.

52. Cheung, C.Y., et al. 2002. Induction of proinflammatory cytokines in human macrophages by influenza A (H5N1) viruses: a mechanism for the unusual severity of human disease? Lancet. 360:1831-1837.

53. Hayden, F.G., et al. 1998. Local and systemic cytokine responses during experimental human influenza $\mathrm{A}$ virus infection. Relation to symptom formation and host defense. J. Clin. Invest. 101:643-649.

54. Fritz, R.S., et al. 1999. Nasal cytokine and chemo- kine responses in experimental influenza A virus infection: results of a placebo-controlled trial of intravenous zanamivir treatment. J. Infect. Dis. 180:586-593

55. Kaiser, L., Fritz, R.S., Straus, S.E., Gubareva, L., and Hayden, F.G. 2001. Symptom pathogenesis during acute influenza: interleukin- 6 and other cytokine responses. J. Med. Virol. 64:262-268.

56. Kobasa, D., et al. 2004. Enhanced virulence of influenza A viruses with the haemagglutinin of the 1918 pandemic virus. Nature. 431:703-707.

57. Rapaport, D., Ovadia, M., and Shai, Y. 1995. A synthetic peptide corresponding to a conserved heptad repeat domain is a potent inhibitor of Sendai viruscell fusion: an emerging similarity with functional domains of other viruses. EMBO J. 14:5524-5531.

58. Lambert, D.M., et al. 1996. Peptides from conserved regions of paramyxovirus fusion (F) proteins are potent inhibitors of viral fusion. Proc. Natl. Acad. Sci. U. S. A. 93:2186-2191.

59. Yao, Q., and Compans, R.W. 1996. Peptides corresponding to the heptad repeat sequence of human parainfluenza virus fusion protein are potent inhibitors of virus infection. Virology. 223:103-112.

60. Wild, C.T., Shugars, D.C., Greenwell, T.K., McDanal, C.B., and Matthews, T.J. 1994. Peptides corresponding to a predictive alpha-helical domain of human immunodeficiency virus type 1 gp 41 are potent inhibitors of virus infection. Proc. Natl. Acad. Sci.U.S. A. 91:9770-9774.

61. Lu, M., Blacklow, S.C., and Kim, P.S. 1995. A trimeric structural domain of the HIV-1 transmembrane glycoprotein. Nat. Struct. Biol. 2:1075-1082.

62. Wild, C., Oas, T., McDanal, C., Bolognesi, D., and Matthews, T. 1992. A synthetic peptide inhibitor of human immunodeficiency virus replication: correlation between solution structure and viral inhibition. Proc. Natl. Acad. Sci. U. S. A. 89:10537-10541.

63. Kilby, J.M., et al. 1998. Potent suppression of HIV-1 replication in humans by $\mathrm{T}-20$, a peptide inhibitor of gp41-mediated virus entry. Nat. Med. 4:1302-1307.

64. Chan, D.C., Chutkowski, C.T., and Kim, P.S. 1998. Evidence that a prominent cavity in the coiled coil of HIV type $1 \mathrm{gp} 41$ is an attractive drug target. Proc. Natl. Acad. Sci. U. S. A. 95:15613-15617.

65. Cianci, C., et al. 2004. Targeting a binding pocket within the trimer-of-hairpins: small-molecule inhibition of viral fusion. Proc. Natl. Acad. Sci. U. S. A. 101:15046-15051.

66. Russell, C.J., Kantor, K.L., Jardetzky, T.S., and Lamb, R.A. 2003. A dual-functional paramyxovirus $\mathrm{F}$ protein regulatory switch segment: activation and membrane fusion. J. Cell Biol. 163:363-374.

67. Hoffman, L.R., Kuntz, I.D., and White, J.M. 1997. Structure-based identification of an inducer of the low-pH conformational change in the influenza virus hemagglutinin: irreversible inhibition of infectivity. J. Virol. 71:8808-8820.

68. Neurath, A.R., Strick, N., Jiang, S., Li, Y.Y., and Debnath, A.K. 2002. Anti-HIV-1 activity of cellulose acetate phthalate: synergy with soluble CD4 and induction of "dead-end" gp41 six-helix bundles. BMC Infect. Dis. [serial online]. 2:6. http://www. biomedcentral.com/1471-2334/2/6

69. von Itzstein, M., et al. 1993. Rational design of potent sialidase-based inhibitors of influenza virus replication. Nature. 363:418-423.

70. Air, G.M., and Laver, W.G. 1989. The neuraminidase of influenza virus. Proteins. 6:341-356

71. Palese, P., and Compans, R.W. 1976. Inhibition of influenza virus replication in tissue culture by 2-deoxy-2,3-dehydro-N-trifluoroacetylneuraminic acid (FANA): mechanism of action. J. Gen. Virol. 33:159-163.

72. Woods, J.M., et al. 1993. 4-Guanidino-2,4-dideoxy2,3-dehydro- $\mathrm{N}$-acetylneuraminic acid is a highly effective inhibitor both of the sialidase (neuraminidase) and of growth of a wide range of influenza A and $\mathrm{B}$ viruses in vitro. Antimicrob. Agents Chemother. 37:1473-1479.

73. Matrosovich, M.N., Matrosovich, T.Y., Gray, T., Roberts, N.A., and Klenk, H.D. 2004. Neuraminidase is important for the initiation of influenza virus infection in human airway epithelium. J. Virol. 78:12665-12667.

74. Hayden, F.G., et al. 1997. Efficacy and safety of the neuraminidase inhibitor zanamivir in the treatment of influenzavirus infections. GG167 Influenza Study Group. N. Engl. J. Med. 337:874-880.

75. Hayden, F.G., et al. 1999. Use of the oral neuraminidase inhibitor oseltamivir in experimental human influenza: randomized controlled trials for prevention and treatment. JAMA. 282:1240-1246.

76. Hayden, F.G., et al. 2000. Oral oseltamivir in human experimental influenza B infection. Antivir. Ther. (Lond.). 5:205-213.

77. Hayden, F.G., et al. 2000. Inhaled zanamivir for the prevention of influenza in families. Zanamivir Family Study Group. N. Engl. J. Med. 343:1282-1289.

78. Monto, A.S., et al. 1999. Zanamivir in the prevention of influenza among healthy adults: a randomized controlled trial. JAMA. 282:31-35.

79. Welliver, R., et al. 2001. Effectiveness of oseltamivir in preventing influenza in household contacts: a randomized controlled trial. JAMA. 285:748-754.

80. Tumpey, T.M., et al. 2002. Existing antivirals are effective against influenza viruses with genes from the 1918 pandemic virus. Proc. Natl. Acad. Sci. U. S. A. 99:13849-13854.

81. Leneva, I.A., Goloubeva, O., Fenton, R.J., Tisdale, M., and Webster, R.G. 2001. Efficacy of zanamivir against avian influenza $A$ viruses that possess genes encoding $\mathrm{H} 5 \mathrm{~N} 1$ internal proteins and are pathogenic in mammals. Antimicrob. Agents Chemother. 45:1216-1224.

82. Leneva, I.A., Roberts, N., Govorkova, E.A., Goloubeva, O.G., and Webster, R.G. 2000. The neuraminidase inhibitor GS4104 (oseltamivir phosphate) is efficacious against A/Hong Kong/156/97 (H5N1) and $\mathrm{A} /$ Hong Kong/1074/99 (H9N2) influenza viruses. Antiviral Res. 48:101-115.

83. Centers for Disease Control. 2004. Update on influenza $\mathrm{A}(\mathrm{H} 5 \mathrm{~N} 1)$ and SARS: interim recommendations for enhanced U.S. surveillance, testing, and infection controls. http://www.cdc.gov/flu/han020302.htm.

84. Commonwealth Scientific and Industrial Research Organization. 2004. CSIRO based drug effective against bird flu. http://www.csiro.au/index.asp? type $=$ mediaRelease $\&$ docid $=$ PrBirdFlu5\&style $=$ mediarelease.

85. McKimm-Breschkin, J., et al. 2003. Neuraminidase sequence analysis and susceptibilities of influenza virus clinical isolates to zanamivir and oseltamivir. Antimicrob. Agents Chemother. 47:2264-2272.

86. McKimm-Breschkin, J., et al. 1998. Mutations in a conserved residue in the influenza virus neuraminidase active site decreases sensitivity to Neu5Ac 2en-derived inhibitors. J. Virol. 72:2456-2462.

87. Varghese, J.N., et al. 1998. Drug design against a shifting target: a structural basis for resistance to inhibitors in a variant of influenza virus neuraminidase. Structure. 6:735-746.

88. Kiso, M., et al. 2004. Resistant influenza A viruses in children treated with oseltamivir: descriptive study. Lancet. 364:759-765.

89. Whitley, R.J., et al. 2001. Oral oseltamivir treatment of influenza in children. Pediatr. Infect. Dis. J. 20:127-133.

90. Moscona, A. 2004. Oseltamivir-resistant influenza? Lancet. 364:733-734.

91. Breese Hall, C. 2001. Respiratory syncytial virus and parainfluenzae virus. N. Engl. J. Med. 344:1917-1928. 